\title{
Arousing a wide range of emotions within educational virtual reality simulation about major depressive disorder affects knowledge retention
}

\author{
Christos Hadjipanayi ${ }^{1,2}\left[\right.$ [D $\cdot$ Despina Michael-Grigoriou ${ }^{1,2}$ (D)
}

Received: 13 June 2020 / Accepted: 30 July 2021 / Published online: 31 August 2021

(c) The Author(s) 2021

\begin{abstract}
The experience of using an educational application, concerning a major depressive disorder simulation, could be anything but pleasant, so the challenges of creating such an application are ample. In this research, the effects of the emotional experience of the players, deriving from the positive ending of the virtual reality (VR) simulation's embedded narrative or the lack of it, are evaluated. Alongside the investigation of a possible link between the emotional impact of the simulation and information retention, the overall effect of the application in relation to VR presence and body ownership is appraised. Thirty participants over 18 years old tested the application, using an Oculus Rift head-mounted display with a joystick, and their data were recorded by a pre- and a post-questionnaire. The 30 participants have been separated into groups of 15 , where the positive ending was accessible to only one of the two groups. The group which experienced the positive ending reported a significant correlation of emotional impact and knowledge retention.
\end{abstract}

Keywords Virtual reality $\cdot$ Game narrative $\cdot$ Affective states $\cdot$ Knowledge retention $\cdot$ Major depressive disorder

\section{Introduction}

The present paper focuses on the fields of virtual reality (VR), affective states, and game design. Computer simulations of real-life situations in combination with gamification elements have an increasingly critical role in many areas of science, engineering, and education (Schultheis and Rizzo 2001; Marsh et al. 2010). This paper attempts to evaluate some qualities of the simulated experience through two different versions of a VR simulation. The difference between the two versions lies in the presence or absence of one extra scene, serving as a hopeful conclusion (positive ending) after experiencing a simulated representation of depression and specifically major depressive disorder (MDD). The simulation encompasses many variables, which are determinant for the user's feeling of contentment toward the overall VR experience and the functionality of the application. The

Despina Michael-Grigoriou

despina.grigoriou@cut.ac.cy

1 GET Lab, Department of Multimedia and Graphic Arts, Cyprus University of Technology, Limassol, Cyprus

2 CYENS Centre of Excellence, Nicosia, Cyprus insight provided from this study could contribute to better understanding the science behind emotional responses elicited by interactive applications due to their capability of promoting emotional priming, which encompasses the subliminal regulation of emotions that determine the state of mind (Rao et al. 2019). The present research explores the potential of interactive applications and their narratives as powerful means of communication and learning.

The meaning of the word "narrative," when used to describe the story of a game (or a gamified VR simulation in this case), differs in some aspects when it is compared to the narrative of more traditional media, like movies and plays (Consalvo 2003). Specifically, game narratives tend to be interactive, nonlinear, and resemble simulations rather than representations of the ongoing events (Qin et al. 2009; Frasca 2013), which is the standard case in more traditional media. Also, the structure of this kind of narrative is twofold; there is the embedded narrative, which provides the player with the context of the game, and the emergent narrative, which depends solely on the player's decisions and actions (Salen et al. 2004). Some researchers and game fans argue that a game narrative focusing on a rich and complex story (embedded narrative) usually interferes with "good" gaming (emergent narrative) (Consalvo 2003). Even though 
game narratives propose the possibility of escaping from the linear, irrevocable events of a film, technological and resource limitations often prevent games from reaching their full potential (Consalvo 2003; Tanenbaum and Tanenbaum 2010). These limitations seem to scale down rapidly as technology advances while providing game designers with fascinating opportunities for novel narrative expressions. Various studies on the game franchise "Mass Effect" detail the increase in the satisfaction of the players when they are given the choice to actively influence the outcome of the narrative (Bizzocchi and Tanenbaum 2012; Moser and Fang 2015). In this regard, sometimes the game may offer an "illusion of choice," a choice that has no actual impact on the outcome of the narrative. The "illusion choice" is perceived by the player as a pivotal point to a narrative outcome because the player is supposedly unaware of the greater picture of the game's narrative structure and therefore the "illusion choice" upholds the same significance for the player as any other major choice in the game (Moser and Fang 2015). The "illusion of choice" is utilized to some extent in the present simulation, as it is considered to be a narratively sophisticated and effective approach for engaging the player with an interactive experience (O'Sullivan 2019).

VR simulations proved to be effective mediators of human experiences, including issues concerning life and death situations. The personal, confessional game "That Dragon, Cancer" presents the story of Ryan (2016) and Amy Green, who lost their son to a rare form of cancer. The game describes how the parents coped with this unfortunate event through their stages of grief (That Dragon, Cancer 2016). Ryan, the creator of the aforementioned game, highlights that videogames allow the player to linger in spaces, even those spaces that exist between scenes, and he finds this property to be exceedingly impactful from a psychological standpoint (Schott 2017). The mediated experiences through VR technology apply to physical aspects as well as mental. Notes on Blindness is another VR game that narrates the story of John Hull, who recorded his daily life experiences while coping with vision deterioration (Oculus n.d.).

Having in mind the examples of "That Dragon, Cancer" and "Notes on Blindness," it is evident that the multimodality of VR applications could transform immaterial personal experiences into forms of self-expression, which aid in expanding the perceptual thinking. Multimodality theory suggests that human experiences are better conveyed the more communication channels are deployed for the production of meaning (Hammond 2009; Kress 2010) and, as a communication theory, it is constituted through the convergence of anthropological, semiological, and educational backgrounds (Kress 2010). In the case of the present study, this expressive potential is partly explored through the scope of multimodal applications on mental health awareness. Multimodality theory tends to take a formalistic approach with little regard to the aspects of content and context, for which it was criticized by Bazalgette and Buckingham (2013). Even so, the narrative produced by this multimodal, meaning-producing process is viewed by the present study from a different perspective, taking into account its instrumental value as a mediator of subjective experience and a carrier of novel knowledge.

\section{Affective states and emotional impact}

The emotional impact referred to in this research is a more convenient wording for addressing the difference between affective states evoked by the players before and after the experience. In psychology, the "affective state" of an individual is characterized by the principal dimensions of valence and arousal. Affective valence is the subjective feeling which results as a (pleasant or unpleasant) consequence to the affect-inducing situation (Harmon-Jones et al. 2013). Arousal is relevant to the activation of the sympathetic nervous system (SNS), which is responsible for the unconscious physiological reactions because of the intensity of the affect (Brodal 2004; Harmon-Jones et al. 2013).

Affective valence and arousal are essential for the study of affective states. Valence, the positivity or negativity of an experience, is a subjective notion that often varies for each individual. Valence is possibly influenced by a personal tendency in focusing on pleasant or unpleasant aspects of an experience (Barrett 1998). Arousal refers to the sensory aspect of incorporating subjective experiences into conscious affective experiences. In other words, arousal relates to the interpretation of affective experiences in semiological terms and the state of alertness they evoke (Christianson 1992; Barret 1998). Feldman sets both valence focus and arousal focus as dimensions of a semantic circumplex, where emotions are categorized as either high or low in accordance with the two dimensions. This categorization mitigates the mapping of emotions and offers an organized theoretical framework (Feldman 1995; Barret 1998). In this study, we use Feldman's categorization in calculating the emotional impact.

Emotions and moods are organized following the intensity of activation in appetitive or defensive reactions. From an anthropological standpoint, affects are cues that motivate relevant actions, which work in favor of survival (Bradley et al. 2001). In cases of emergency, actors become more attentive because their senses grow sharper, and this neurological reaction persists even if the emergency is just in a virtual simulation (Chittaro et al. 2014). Motivational intensity theory stresses that the feasibility and importance of achieving a goal are determinants of the energy invested for its success (Richter 2013). The scientific literature supports the association between motivational intensity and affective 
states, further suggesting that there is a link between cognitive and affective processes that is responsible for defining goals and allocating resources (Bradley et al. 2001; HarmonJones et al. 2012; Villani et al. 2009). Motivational intensity could be characterized as "the urge to move toward or away from a stimulus" (Harmon-Jones et al. 2013). Regarding the present research, motivational intensity is utilized in the form of "overcoming a depressive episode."

Empathy is a crucial factor in the assessment of the narrative's emotional impact. The phenomenon of empathy in its broader sense is the cognitive, intersubjective notion of going through the observed experiences of another person, thus understanding them better (Davis 1983; Decety and Hodges 2006; Gallagher 2012). Some people have the capacity to empathize more than others, as empathy level could be determined by specific situations (situational empathy) but also from characteristic traits of individuals (dispositional empathy) (Muller et al. 2017). Dispositional empathy is influenced by easily identifiable characteristics, such as gender (Davis 1980), but also from covert psychological deviations, such as personality traits of the dark triad (Schimmenti et al. 2019). VR perspective-taking, the use of VR technology and immersive technology for conveying the experiences of others, is considered to be an effective method for eliciting empathy (Christofi et al. 2020). However, VR perspective-taking is only a step to understanding what is like to walk in the shoes of another person. Empathy operates best while inside a situation, which provides a concrete context with direct relation to affective states. More explanatory, empathy elicits emotional responses, which are directed from one person to another, especially between people who experience first-hand the element of hardship. Empathy's connection with narrative stems from the familiarity of the empathizer to another individual and the vicariousness of the experienced situation (Ghallagher 2012). Therefore, in our simulator, the participant receives the experience from the first-person perspective of a virtual person, who suffers from major depressive disorder.

Before elaborating more on the present simulation, the affective aspect of virtual environments (VEs) must be addressed. Some researchers particularly address the impact of immersion and emotion on the sense of presence (Baños et al. 2004). Their research tests the extent to which the emotional aspect of the content can enhance the illusion of virtual presence in a VE, through three different media. For each medium (PC monitor, HMD, and big screen), two groups of participants were formed, and each was exposed in content, which differed slightly in comparison. The VE is representing a park, which appeared to have a colorful and sunny environment for the control group, but for the experimental group, the environment appeared to be sunless, melancholic, and deserted, thus evoking a feeling of sadness. The HMD, as a medium, scored lower than the PC monitor and the big screen, regarding the subjective sense of presence in the "sad" VE despite HMD consisting of a fully immersive $360^{\circ}$ system. The researchers associate this result with the negative effects often caused by HMDs such as dizziness, nausea, and disorientation so the findings remain slightly inconclusive (Baños et al. 2004). Later studies on the link between presence and emotions conclude that there is a bidirectional influence between the two, which is directly proportional (Riva et al. 2007).

However, the link between presence and emotions in VR remains unclear, despite the revolutionary contribution of VR technology in clinical psychology (Diemer et al. 2015). A popular theory, which is rooted in the theory of motivational intensity and aspires to explain the link between presence and emotions in VR, supports that affects of high arousal activate high alertness, which automatically leads to a sharper sense of presence (Freeman et al. 2005). By contrast, Baños et al. argue that "high-quality sensory data" and "good, convincing storytelling" are the key aspects of presence, as they discovered positive correlations between virtual presence and low arousal affects, such as relaxation (2008). The theoretical model of Seth et al., instead of focusing on the intensity of emotions, reports predictive coding to be interconnected with presence, in a way that the precision of the perception of the physiological condition of the body (interoceptive predictive signals) is a central factor in inducing the sense of presence (2012). The salience of emotional affects in causing a sense of presence in VR is still under scrutiny.

The study of Villani and colleagues is relevant to the present study since it focuses on VR as an affective medium. Villani et al. highlight the importance of "media content" and "media form" when it comes to affective experiences in VR (2009). "Media content" refers to what the present study already defined as "narrative" and "media form" refers to the setting of the VE, which inevitably provokes emotional responses of subjective nature. As the same study suggests, the media form plays a critical role on a visceral level, while media content has a more reflective impact. Both media form and media content are found to be essential for creating an affective experience, as they also influence the sense of presence. Villani et al. define presence in the technical sense of "media presence," as "a function of the experience of a given medium" (2009). A common problem of the use of VR technology in research is the diversification of the definition of presence, which complicates the operationalization of presence and its components (Diemer et al. 2015). The present study is using a definition of presence that is more relevant to its neuropsychological sense that associates presence with emotion (Slater 1999). According to Slater, presence is "form" and emotion is "content." Emotion could be used to verify whether there is presence or not and this 
dynamic distinguishes the concepts of emotion and presence as separate (2003).

The definition of presence in this study refers to the sense of "being there" (Slater 2009). Presence and body ownership are referred as VR illusions. The VR illusions, which are observed in this study, derive from the multisensory input provided by the VR system. Body ownership refers to the perceptual illusion that a virtual avatar is the body of the user, despite the physicality of the user (Hasler et al. 2017). Furthermore, this illusion refers to the unique sensation of owning a physical body, which transcends the physical plane and transfers to the virtual (Maister et al. 2015).

In this study, the convergence of VR illusions (presence, body ownership) and affective states, which share a connection to behavioral change as already stressed, is also examined. Users of avatars tend to adopt attitudes, which correspond to the attributed dispositions of their avatars, pointing out that virtual avatars have a considerable attitudinal and behavioral impact. For instance, racial bias is reported to diminish as an after-effect of embodying the avatar of an outgroup member (Maister et al. 2015; Banakou et al. 2016; Hasler et al. 2017). A set of studies by Jun et al. supports the positive correlation between body ownership and affective states. It is found that an angry-looking avatar induces negative valence and a happy-looking avatar induces positive valence (2018). The studies mentioned are acknowledging the fact that similar studies should be carried out within the domain of psychological disorders, such as depression (Jun et al. 2018). The proposed application of these similar studies is within the context of behavioral activation for the treatment of depression, whereas the present study is more relevant to learning about depression, acknowledging, and preventing it.

\section{Major depressive disorder}

Major depressive disorder (MDD) is mainly used as a vehicle for the conduction of this research, but it is also a major focus of this study. The content of the simulation is heavily influenced by MDD; thus, a compendious citation of this psychological disorder is needed. Also, the need for education around MDD and other depression disorders is eminent, as previous studies indicate that the community ranks mental illnesses as less threatening than other pathology issues, like addiction and physical illness (Highet et al. 2002). More recent studies show that preconceived notions about depression, its treatment, and help-seeking practices for psychological well-being are mostly ethnicity based. For example, black African women were found to underrate the severity of the consequences caused by depression and consider helpseeking for depression as unnecessary significantly more than British women (Brown et al. 2011). African-American men were also found to perceive depression as synonymous with sadness and consider help-seeking for depression as unnecessary and ineffective (Hudson et al. 2018).

A different study reveals that online literacy on depression is capable of substantially educating and reducing personal stigma regarding depression but the general views of how society stigmatizes people with depression remain quite discouraging (Kiropoulos et al. 2011). The exact causes of depression are still a mystery, even though current scientific findings point toward biological and social factors therewithal (American Psychiatric Association [APA] 2013). Psychosocial stress is considered to be the public's most perceived cause of depression (Lauber et al. 2003). Attempts to minimize the negative stigma surrounding depressive disorders and increase public awareness on MDD symptoms and mental health issues should be more broadly encouraged.

Some MDD symptoms manifest more frequently than others. Symptoms of MDD are presented and discussed in this paragraph. The most common symptom of MDD is dejected mood. The patient has a fleeting feeling of dysphoria when depression is mild, but when depression is moderate or severe, dysphoria becomes progressively more persistent and painful (Beck and Alford 2009). Another symptom of MDD is the erosion of self-confidence. Almost every MDD patient experiences self-disappointment which could escalate into self-loathing in severe cases (Beck and Alford 2009; Schimelpfening 2018). The irrational feeling of worthlessness is often accompanied by the feeling of irrational guilt (Schimelpfening 2018). Patients, who suffer from severe MDD, experience difficulties in carrying out daily activities, because they are tortured by nihilistic delusions, sleep and appetite disturbances, as well as aversion toward activities they once enjoyed (Beck and Alford 2009; Schimelpfening 2018). The ideas of death and suicide seem compelling to MDD patients. They often resort to perilous behaviors, which could end up being fatal (Beck and Alford 2009). Lastly, some physiological symptoms of MDD include motor retardation, slow speech, and outbursts of lamentation (Caligiuri and Ellwanger 2000; Beck and Alford 2009; Schimelpfening 2018).

The symptoms of MDD mentioned above have been transferred to our simulator through the narrative and by integrating related information to a number of points of interest within the VE, which are discussed in detail in section "The scenario".

\section{Motivation}

The development of educational content in the domain of mental illness contributes to the discovery of important insights, which in many instances could prove to be lifechanging for individuals, regardless of their current mental 
state. Especially after the rise of the Medical Humanities movement, individual experiences in the form of narrative, relating to mental illness, are deemed to be remarkable artifacts of academic value (Green and Myers 2010). The acknowledgment of these narratives as noteworthy not only affirms the intersubjective struggles of mental illness but also helps in addressing mental illness and explore its effects. The VR application of the present study incorporates narratives of ex MDD sufferers, who wish to remain anonymous, supported by data deriving from the scientific literature and DSM-5 (American Psychiatric Association [APA] 2013).

VR is specifically chosen as a suitable medium for this application because of the level of interactivity it allows. Ryan (2001) highlights the capability of VR to synthesize interactivity and immersion for the production of meaningful experiences. This combination is characterized by Ryan (2001) as "the ultimate goal of art." Apart from the ability of VR to immerse users in narratives, VR is also chosen because of its educational capabilities. VR offers controlled environments, adaptable to a plethora of learning subjects (Diemer et al. 2015). Most importantly, the highly immersive and game-like approach of VR applications covertly transforms learning into enjoyable experiences (Freina and Ott 2015). This final remark is most disconcerting for the present application, as the experience portrayed by MDD is intrinsically meant to be the opposite of enjoyable.

The complications of this educational application, being fundamentally depressing, probably go beyond the mentioned aesthetic aspects. The cause of repulsion toward the game narrative and its depressing content may interfere with the informational capacity of the application, as suggested by Bower et al. (1981). Performance in cognitive functions (e.g., the act of categorizing words according to their perceived pleasantness) is found to be highly influenced by affective states (Bower et al. 1981; Johnston and Dark 1986). Furthermore, the congruence of emotions, such as sadness, between fictional characters and readers was found to be beneficial for information retention (Johnston and Dark 1986). However, the present VR application, which narratively relays educational information, is primarily directed toward individuals who are in a "healthy state of mind," as opposed to the depressed avatar that they are encouraged to identify with. Admittedly, the informative aspect of such simulation may lack the arousal intensity that is found to benefit information retention (Chittaro et al. 2014). In this study, the effect of a positive resolution in the narrative, namely a happy conclusion, is deployed to ameliorate the dissonance between the emotional states of user and avatar, act as a reward, and recompensate for the lack of arousal intensity. The findings of the present study could provide some insight regarding educational simulations, which could potentially lead to the improvement of future educational simulations and their corresponding narrative approaches.

\section{Research question}

The research question focuses on the differences in the affective states and information retention between two narratives of which one of them is purely depressing, while the other is still depressing but has a happy ending. The first narrative is assumed to evoke negative emotions, while the latter is assumed to evoke a wider range of emotions, both positive and negative. More explanatory, the first condition promotes an experience that is admittedly repulsive to the memory due to its purely negative connotations, in contrast to the latter, which aspires to alleviate the said unpleasantness. Because the participants of the experimental group are encouraged to seek help, it is speculated that the expectation and hope of receiving help would stimulate the participants' reward system and recompensate for the simulation's inherently low arousal level. On the basis of the motivational intensity theory (Richter 2013), the positive ending is integrated as a reward for achieving the assigned goal. Therefore, knowing that access to a positive ending should be available, is considered to evoke a higher motivational intensity, as participants are expected to feel compelled to access the positive ending. This positive ending is of course intended to be as objectively positive as possible. A positive ending inevitably affects the overall valence of the experience for some participants. The experience of using the MDD simulation is assessed by the parameters of knowledge retention, emotional impact, presence, and body ownership, while taking into consideration the interplay between them and other variables, such as gender and dispositional empathy. Research question: Does increased motivational intensity have a positive effect on the experience of using the MDD simulation? It is hypothesized that the inclusion of the positive ending will contribute to a more emotionally impactful and rewarding experience, which will, in turn, result in higher information retention. Hypothesis 1: Increased motivational intensity has a positive effect on emotional impact. Hypothesis 2: Increased motivational intensity has a positive effect on knowledge retention. Hypothesis 3: Increased motivational intensity has a positive effect on the VR illusions of presence and body ownership.

\section{The MDD VR simulator}

Focusing on an educational simulation about MDD with gamification elements, in this research, the emotional impact between two similar scenarios is evaluated. What the two scenarios have in common is that the user enters the $3 \mathrm{D}$ 
avatar of an MDD patient through a first-person perspective, inside an imaginary bedroom, and the user is encouraged to explore the surroundings while discovering the pathology of MDD. After exploring the bedroom, the narrative, in only one of the two scenarios, offers the player the relief of shifting toward a psychologically healthy perspective (positive ending). The positive ending refers to the part where the MDD patient was successfully treated after seeking help. The virtual environment changes to reflect the psychological state of the MDD patient.

The interaction of the user with the points of interest in the virtual environment is accomplished by using a gaze control mechanic (Fig. 1). This concept has been borrowed from Hadjipanayi and Michael-Grigoriou (2020). The animated avatar of the depressed woman is situated in the middle of the bedroom, while the user inhabits its body. The avatar's body orientation is synchronized with the angle of the head mount display. For moving toward any direction, the use of a joystick is required. The application begins with the woman explaining her negative experience with MDD for approximately $30 \mathrm{~s}$, as part of the embedded narrative. The information regarding the thoughts, emotions, misconceptions, and visceral reactions of the depressed person in the scenario was accumulated through the corresponding scientific bibliography and personal narratives of MDD patients, who recovered from a depressive episode. The

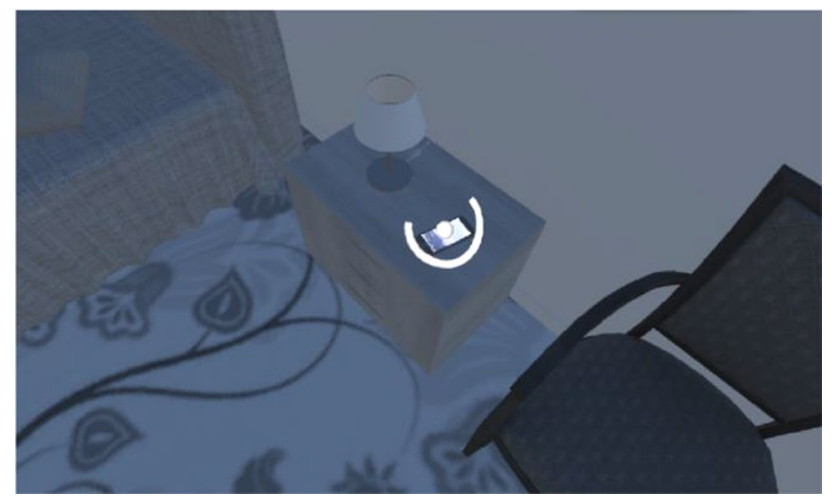

Fig. 1 The player is exploring the environment by using the gaze control mechanic personal narratives were collected through testimonies of consenting adults, who wish to remain anonymous.

\section{The scenario}

After the introductory monologue of the MDD patient, the users of the simulation are free to explore the VE, by using the MDD patient as an avatar. It should be noted that, during the introductory part, the MDD patient acknowledges that something is "wrong" with her, but she is unable to specify her condition. She is faced with irrational guilt trips, arising from her false assumptions. Inside the room, six points of interest can be found, whose discovery unlocks the final part of the embedded narrative, namely the positive ending (in the case of the experimental group only). There is a seventh point of interest, the door, whose activation is pivotal for the experimental group and optional for the control group. For the experimental group, the door serves either as an indication that the participant has not discovered all six points of interest yet or otherwise as a passage to the final scene. In the first case, the MDD patient is making this indication evident by expressing the need to stay for a little longer in her room, for her to muster the courage to go on.

The six objects serving as points of interest in the room are the bed, the library, the mirror on the wall, the empty plate on the desk, the smartphone on the bedside table, and the ceiling lamp (Fig. 2). All of them unlock a different narrative, shedding light on aspects of MDD. The bed highlights the state of lethargy and the irregular sleeping patterns, from which MDD patients suffer. The library shelves are filled with books and plushies, many of which are not orderly aligned. Plus, some of the books are lying on the floor. According to the MDD patient, she attempted to re-engage with activities, which used to bring her joy, but soon she realized that her attempts to garner joy were vain, as expected from a severely depressed individual. The mirror on the wall reflects the idol of the avatar, further contributing to the sense of the player's bodily illusions in the VE, namely the senses of self-location, agency, and body ownership that constitute the sense of embodiment, which is the influence of
Fig. 2 The VE with some of the points of interest, such as the library, the bed, and the empty plate, through the lens of MDD (left) and without the influence of MDD (right)
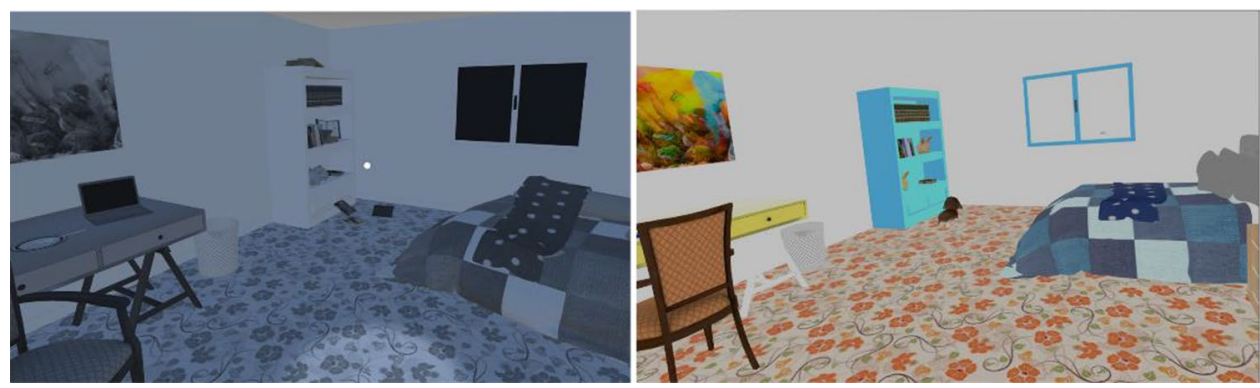
the bodily sensation on perception (Kilteni et al. 2012; Lopez et al. 2008) (Fig. 3). As another point of interest, the mirror offers the opportunity for self-evaluation to the MDD patient. The decay of self-confidence and the feeling of self-loathing are prominent, through her words. The empty plate activates the narrative about the change of her eating patterns as well as her tendency to self-harming behavior. The several missed calls on her smartphone urge her to express her repulsion toward any social activity. One highlighted causality of this repulsion is the fact that the MDD patient is aware of her defective mental condition, so she feels uncomfortable projecting it on others by being close to them. Lastly, the MDD patient is imagining a noose hanging from the ceiling lamp, indicating her suicidal impulses. If the door point of interest is activated before all the other points of interest are discovered, the MDD patient thinks that she needs some more "time" to muster the courage to get out of her room. The MDD patient is thereby expressing her intention to fight against the urge to succumb into MDD at some point in time while the participant is given the chance to linger into the room and discover all the points of interest before moving on.

The positive scene, showed at the end of the narrative only in one of the two versions of the simulator, has almost the same design as the first scene but with many changes on the brighter side of the spectrum (Fig. 2). The bedroom of the second scene looks bright, colorful, tidy, and sunlit, unlike how it seemed in the previous scene (Fig. 2). The background music changes from dramatic to happy. Plus, a pet-dog is found strolling around the room. The body posture of the avatar and the vigor of the avatar's leap improve significantly, highlighting the escape of an impaired state of motor functions. There is no interactivity involved with the objects of the room in this scene. The application closes as soon as the woman concludes her story, on how she was able to have her MDD treated. A mindset change and the rejection of her misconceptions on MDD are also apparent. A video demonstrating the scenario of the VR application is given in the submitted supplementary materials.

\section{The avatar}

The only available avatar and the assigned voice clips found in the MDD simulation are implying the representation of a female character. It could be reasonable for one to assume that the female participants are much more likely to feel intimate in their virtual (female) body and gain precedence over dispositional empathy, compared to men, but the reasoning behind using a female avatar is more complicated. Dispositional empathy is said to be a lot more prevalent in women than men since women tend to display higher scores on the IRI scale used for measuring the dispositional empathy of participants (Davis 1980). The polarized results of the empathy scale could serve as an indication that people tend to act according to gender stereotypes and culturally influenced roles. Baron-Cohen's theory of the extreme male brain (2002) suggests that men tend to "systemize," while women tend to "empathize" more because of the biological wiring of their brains. The theory of the extreme male brain, as well as similar gender-based theories, is considered to be debatable for some researchers because of neuroethical implications (Fine 2008). Despite the validity of significant neurobiological differences between genders, the evidence that people are instilled with the tendency to act upon socially constructed stereotypes remains. The study field of virtual avatars has the extensive literature on aspects related to gender stereotypes and a branch of related studies has the Proteus effect on its basis. The theory of the Proteus effect suggests that users of avatars in virtual environments are imbued with the assumed qualities and abilities of their respective avatars, and these abilities also relate to the socially constructed gender stereotypes (Lee et al. 2014). The utilization of stereotypes for enhancing specific abilities of VE users, also known as virtual stereotype lift, applies to a vast range of tasks, from math solving to expressions of aggression (Lee et al. 2014; Walton and Cohen 2003). Even though the virtual stereotype lift becomes stronger when the avatar competes directly against an outgroup (Walton and Cohen 2003), in this study a female avatar is used non-competitively to promote empathy to every participant regardless of gender.
Fig. 3 The player is watching the avatar through the mirror with the influence of MDD while having a depressed disposition (left) and without the influence of MDD (right)

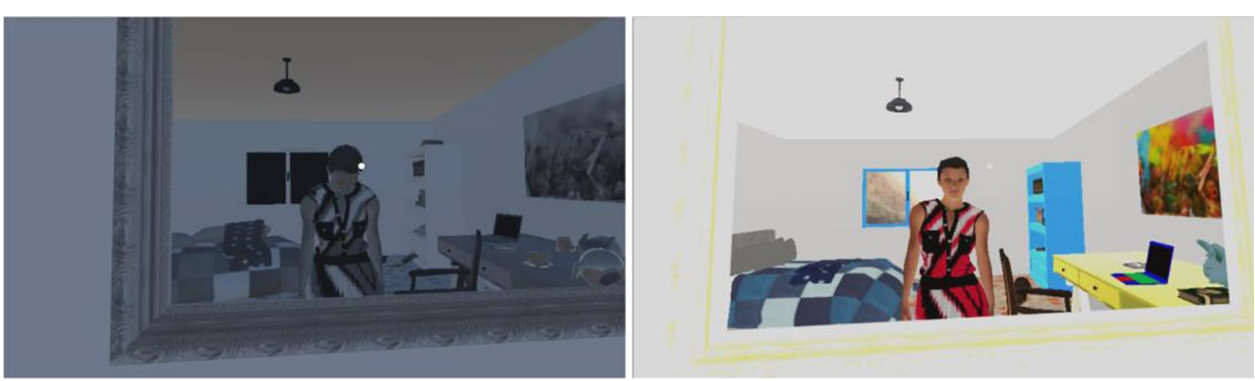




\section{Research methodology}

\subsection{Participants}

Adults over 18 years old were the population of this research, in which the sample was selected using convenience sampling. A total of 30 participants, 15 males and 15 females, volunteered to take part in the experiment.

\section{Procedures}

Participants were exposed to a gamified VR simulation. Each participant experienced the simulation from the firstperson perspective view of the virtual body of an avatar, who was representing a person suffering from a depressive episode. The experimental group refers to the group in which the participants had access to the positive ending, in which the participants were led to anticipate that an incident will occur after the exploration of the first scene. By contrast, participants of the control group were unable to proceed to the positive ending after exploring all the points of interest found in the VE and the chance of an incident occurring after the exploration of the first scene was left unclear. The following paragraphs explain the procedure in more detail.

Firstly, participants from both groups were given an overview of the study and were requested to sign a consent form. Then, they were further informed about the procedure of the experiment, in a quiet and secluded office. Before getting the virtual experience, the participants underwent the pre-tests as described below. Afterward, they were fitted with the VR equipment to experience the virtual narrative of the MDD simulator. They were given some instructions about the control of the interaction with objects in the VE. Within the VE, the participants were invited to the virtual bedroom, while given the role of an MDD patient and allowed to explore the surroundings with the assigned goal of locating all the points of interest, which contain information on MDD.

The above procedure was common for the participants of both groups. However, before the experiment and after the participants consented to participate in a simulation about MDD, the participants of the experimental group (only) were led by the research facilitator to anticipate that an incident will occur after the exploration of the first scene. Specifically, the research facilitator encouraged the participants of the experimental group to keep reaching out for help until they succeed. This encouragement to seek out help is the only procedural factor that differentiates the experimental and the control groups. The participants of the control group received no encouragement prior to the experiment and they were simply instructed to explore the simulation.
After the participants of the control group discovered all the points of interest in the virtual environment, they were informed by the research facilitator that they successfully completed the task. After the participants of the experimental group discovered all the points of interest in the virtual environment, they were not prevented from searching for more clues to help them reach out for help. This action was eventually leading the participants of the experimental group to the re-activation of the door point of interest. By doing so, the positive ending was being triggered, and then, the relevant scene was being displayed to the participants, to inform them that the task was successfully completed. Lastly, the participants of both experimental and control groups were removing the VR headset and completing the post-test.

\section{Materials}

The educational simulation about MDD was designed from scratch for this research, and it was created using the Unity 3D game engine. All the deployed assets have been incorporated with regard to legal or ethical restrictions. The human 3D avatar and her animation sequences were downloaded from www.mixamo.com. The rest of the 3D models, including the virtual mirror, were downloaded from the Unity Asset Store and some were created using the Autodesk Maya modeling software. The setup of the simulation was made with the assistance of tools provided by game developers online (Unity Asset Store 2017). The voice clips used in the simulation were recorded by a consenting adult in the Greek language. The music tracks, which play throughout the scenes, were downloaded from ashamaluevmusic.com (AShamaluevMusic 2017). The programming language used for the scripting is $\mathrm{C \#}$. The application supports the use of the Oculus Rift CV1 hardware set and Xbox Controllers for navigation. The experiment was carried out using a PC with an Intel Core i7-6700HQ 2.6 GHz CPU, 16 GB RAM, and an NVidia GeForce GTX 10606 GB GPU. The setup included the Oculus Rift (CV1) head-mounted display (HMD) with $2160 \times 1200$ resolution $(1080 \times 1200$ per eye), $110^{\circ}$ field of view and $90 \mathrm{~Hz}$ refresh rate for $3 \mathrm{D}$ immersive viewing, head rotational and positional tracking, and provided spatialized audio.

\section{Measures}

This is a quasi-experimental research, following a pre-test/ post-test design and a between-subjects approach.

The participants of both groups underwent a series of the same tests. Before the experiment (pre-test), they completed questionnaires about demographic data (DemQ), a Knowledge Questionnaire (KnQ) with questions related to 
the major depressive disorder, the Discrete Emotions Questionnaire (DEQ), which contains a set of affects, and the Interpersonal Reactivity Index (IRI) test to measure their level of empathy as defined by Davis (1980). After the VR experience, participants of both groups answered the exact same questionnaires about knowledge (KnQ) and emotions (DEQ) as they were undertaken at the pre-test phase. Additionally, questions related to their emotional valance and VR illusions experiences (ExpQ), questions about the application functionality (VRFuncQ), and some other open-ended questions were completed. More details for each questionnaire are provided in the following paragraphs.

Pre-tests required the demographic data (DemQ) of the participants, their current knowledge, and their interest in knowing about MDD. A small assessment followed (KnQ), which clarified the level on which the participants could differentiate the symptoms of MDD and common sadness. KnQ was created based on the information of MDD symptomatology, that the application attempted to convey. This test included seven items and was repeated after the interventions. The items corresponded to either MDD or common sadness. Understandably, symptoms of depression and sadness often overlap so the participants were advised to mark also as depressive (true) the symptoms which presumably apply for both MDD and sadness.

Next, a series of questions requires the current mood of the participants. The Discrete Emotions Questionnaire (DEQ) is a tool based on the theories of Ekman, Izard, and Panksepp, who argue on the concept of "basic emotions." The attempt to organize emotions in categories facilitates the breakdown of emotional experiences and avails research studies on relevant subjects (Ekman 1992). Basic emotions refer to the "raw materials" of the rest of the emotions, which could be empirically distinguished by humans (Ekman 1992; Izard 1992; Panksepp 2004). Each of these researchers presents different sets of emotions as basic, but some of these emotions, such as happiness, sadness, anger, fear, and disgust, were commonly used within most studies. Therefore, in our study, we adopted these five affects as our basic emotions. DEQ was perceived as an adequate tool for this study because of its directness and the flexibility it offers in evaluating emotional impact (Harmon-Jones et al. 2016). DEQ was completed before and after the interventions on a five-point Likert scale. The range of each emotion was calculated by subtracting the participant's post-value from the pre-value. The overall emotional impact was determined by the average of the emotional fluctuations caused by the interventions.

Davis's empathy questionnaire was used for the measurement of dispositional empathy. The Interpersonal Reactivity Index (IRI) measures perspective taking, fantasy, empathic concern, and personal distress as the four components of empathy, the level in which the individual reacts to the observed experiences of another (Davis 1980). The IRI, which was measured on a five-point Likert scale, was translated and validated in the Greek language (Tsitsas et al. 2012). Empathy is deemed to be a baseline for ensuring that the two groups have the same capacity of empathizing with the disposition of the virtual avatar, thus allowing a valid comparison between the emotional impact of any group.

The post-test assesses a multitude of factors essential for the measurement of the narrative's emotional impact. The sense of presence and body ownership is measured after the experiment to measure the level and the congruence of modality on which the participants felt the illusions in the VE (Slater 2009; Steed et al. 2018). The adopted questions of presence and body ownership, taken from the established SUS questionnaire (Slater and Usoh 1993; Slater and Steed 2000), have been used in several other studies (Banakou et al. 2016; Hasler et al. 2017; Steed et al. 2018). Additionally, the post-test encourages participants to leave feedback regarding their VR experience with MDD, through open-ended questions. One closed-ended question requires a pleasant/unpleasant rating on the whole experience, for grouping participants according to their experienced valence. Notably, the application will be considered as a successful educational tool if the participants tend to score higher on the post-test KnQ than the same pre-test KnQ. Descriptive items of the measurements are found in Table 1.

\section{Results}

Statistic tests were used for measuring the differences between groups. Eight males and seven females participated in the experimental group, while seven males and eight females participated in the control group. From the 15 participants of the experimental group, four of them perceived to have an unpleasant experience, despite the effect of the positive ending. Similarly, from the 15 participants of the control group, five of them perceived to have a pleasant experience, despite the absence of the positive ending. The ratio between the positive and negative valence of the two groups is congruent with the expected outcomes.

Dispositional empathy (IRI questionnaire), measured in both groups before the VR experience, was found to have almost the same mean among the experimental and control groups. Independent samples $T$ tests show that there was not any significant difference between the empathy (IRI) of the experimental group $(M=3.53, \mathrm{SD}=0.31)$ and the control group $(M=3.55, \mathrm{SD}=0.34), t(28)=-0.14, p>0.05$, and this offers a desirable balance distribution of participants between the two groups, thus allowing for valid comparisons of emotional differences after the VR experience. Also, no significant difference was found between the dispositional empathy (IRI) of the group, which perceived to 
Table 1 Samples of questions in pre- and post-questionnaires of both groups

Pre-test (only) Measurements (Sample Questions)

Demographics Questions (DemQ) (Categorical)

$$
1
$$

2

3

Demographics Questions (DemQ) (Seven-Point Likert Scale)

4

5

Interpersonal Reactivity Index (IRI) (Five-Point Likert Scale)

1

2

3

4

5

Pre- and Post-Measurements (Sample Questions)

Knowledge Test on the Symptoms of Major Depressive Disorder (KnQ)

Please indicate which of the following items fall under the symptomatology of depression (major depressive disorder) (true/false)

1

2

3

4

5

6

7

Discrete Emotions Questionnaire (DEQ) (Five-Point Likert Scale)

Please rate how you feel right now

$$
1
$$

2

3

4

5

Post-test (only) Measurements (Sample Questions)

1

2

3

VR Experience Questionnaire (ExpQ) (Seven-Point Likert Scale)

1

2

3

4
Age

Gender

Level of education

How would you rate your level of knowledge to depression (major depressive disorder)?

How interested are you in knowing about depression (major depressive disorder)?

I try to look at everybody's side of a disagreement before I make a decision

I sometimes feel helpless when I am in the middle of a very emotional situation

When I see someone who badly needs help in an emergency, I go to pieces

Being in an intense emotional situation scares me

After seeing a play or a movie, I have felt as though I were one of the characters

Unjustifiable feelings of self-blame (true)

Anhedonia (inability to feel pleasure) (true)

Prolonged loss of productivity (true)

Inconsequential to self-esteem (false)

Emotional numbness (true)

Subsiding in due time without treatment (false)

Suicidal ideation (true)

Happiness

Sadness

Anger

Fear

Disgust

\section{Self-Reported Questions on the Functionality of the Application (VRFuncQ)}

How informative was the application about depression (major depressive disorder) compared to what you already knew about it?

Did you find the amount of information about depression (major depressive disorder) enough for you to form a clear understanding of the subject?

How much did the narrative pique your interest?

To what extent did you feel that you and the body of the avatar belonged both to you and a woman with MDD during your virtual experience?

How physically natural was your interaction with the virtual environment?

To what extent were there times during the experience when the virtual environment was the reality for you?

During the experience, which was stronger on the whole? Your sense of being in the virtual environment or the real world? 
Table 1 (continued)

5

VR Experience Questionnaire (ExpQ) (Categorical)

6

Feedback about the Simulation (Open-ended Questions) regarding:

1

2
When you think back about your experience, do you think of the bedroom more as images you saw, or more as somewhere that you visited?

Perceived Valence of Overall Virtual Experience (pleasant or unpleasant)

Functionality

Content have a pleasant experience $(M=3.45, \mathrm{SD}=0.36)$, and the group, which perceived to have an unpleasant experience $(M=3.64, \mathrm{SD}=0.25), t(28)=-1.57, p>0.05$, while using the simulation.

Dispositional empathy (IRI) was correlated with the affective arousal of the participants of the control and experimental groups before and after the experimental phase. Affective arousal was calculated as the average of all the affect measures of the DEQ, which were collected before and after the experiment. Before the experimental phase, it was found that for the experimental group, there was a nonsignificant correlation between dispositional empathy and affective arousal (pre-DEQ), $r(13)=0.28, p>0.05$, and a significant correlation between the two for the control group, $r(13)=0.66, \mathrm{p}<0.01$. The opposite became true after the experiment, where it was found that for the experimental group, there was a very significant correlation between dispositional empathy and affective arousal (post-DEQ), $r(13)=0.8, p=0$, and a nonsignificant correlation between the two for the control group, $r(13)=0.23, p>0.05$.

The emotional impact of the MDD simulation between experimental and control groups was measured. Emotional impact was calculated as the average of changes (before and after the VR experience) of all the affects of the DEQ questionnaire. All the affects were measured before and after the experiment to ensure the validity of the assessments. The Kolmogorov-Smirnov and Shapiro-Wilk tests of normality reported Disgust for being a constant variable, which remained unaffected throughout the experiment, so Disgust was discarded from the analysis. As for the rest of the affects, Happiness and Sadness were found to check the normality assumptions, contrary to Fear and Anger, so one-sample T-test and Wilcoxon sign-rank test were used to analyze each set after the experiment, respectively. Happiness had a significant reduction in both experimental group $(M=2.33$, $\mathrm{SD}=1.29), t(14)=10.86, \mathrm{p}=0$, (reduction $=0.66)$, and control group $(M=2.87, \mathrm{SD}=1.12), t(14)=9.86, p=0$, (reduction $=0.33)$. Similarly, sadness increased significantly for both experimental group $(M=2.93, \mathrm{SD}=1.28), t(14)=8.87$, $p=0$, (increase $=1.26)$, and control group $(M=2.80$, $\mathrm{SD}=1.08), t(14)=10.01, p=0,($ increase $=0.73)$. No significant change in Fear or Anger was observed in either group. Fear had an almost equal increase in both groups ( 0.27 for the experimental and 0.34 for the control). Anger had a slight increase (0.26) in the control group only. The above results suggest that the overall emotional impact was greater in the experimental group, as the range of emotions before and after the experiment was larger for the experimental group compared to the control group.

The perceived valence of the experience of each participant (measured through ExpQ) separates the participants in the groups of "perceived pleasant" and "perceived unpleasant" experience, regardless of the experience of the positive ending or the lack of it. A significant difference between "perceived pleasant" and "perceived unpleasant" experience groups is that for the "perceived pleasant" group, the mean range of sadness was significantly wider $(M=1.06, \mathrm{SD}=1.48)$ than the mean range of sadness from the "perceived unpleasant" group $(M=0.92, \mathrm{SD}=0.82)$, $t(24.08)=0.31, \mathrm{p}<0.05$. Specifically, the mean of sadness for the "perceived pleasant" group before the experiment $(M=1.63, \mathrm{SD}=0.95)$ increased after the experiment $(M=2.69, \mathrm{SD}=1.30)$, and the same applies for the "perceived unpleasant" group, before the experiment $(M=2.14$, $\mathrm{SD}=0.86)$ and after the experiment $(M=3.07, \mathrm{SD}=0.99)$.

The participants' confidence in their knowledge on depression from DemQ on a scale from 1 to 7 is found to be above average $(M=4.5, \mathrm{SD}=1.5$, Median $=5)$, so it was expected that MDD knowledge before and after the experiment will not change significantly because of a ceiling effect. Mann-Whitney $U$ test was used to examine the educational capacity of the application. The knowledge about the symptoms of MDD, measured via the knowledge assessment (pre-KnQ) before the use of the application, is found to be almost the same for the experimental group and the control group $(U=80, p=0.16)$. Knowledge about the symptoms of MDD, measured via the knowledge assessment after the use of the application (post-KnQ), was also found to be almost the same between the two groups $(U=87.50, p=0.25)$. None of the two conditions were causing significantly higher information retention. Regardless of the possible ceiling effect observed, one could suggest that there was a slight 
improvement in the participant's knowledge test score about the symptoms of MDD (KnQ) in both experimental and control groups, by comparing the scores of the tests ( $\max$ score $=7)$ before $(M=5.53, \mathrm{SD}=1.45)$ and after $(M=6.10$, $\mathrm{SD}=1.29)$ the use of the application. Of note, this difference cannot be generalized since it is not significant.

Spearman's rho indicates that the emotional impact (DEQ) of the simulation correlates positively with the post-knowledge test on MDD symptoms (post-KnQ) on a significant degree for the experimental group only $(\rho=0.60, p=0.01)$ (Fig. 4). No significant correlation was found between emotional impact and pre-KnQ for the experimental group. As for the control group, significant correlations regarding emotional impact were found neither for pre-KnQ nor for post-KnQ.

The application has a satisfactory performance (VRFuncQ) since the participants rated its overall performance $(M=5.95, \mathrm{SD}=0.71)$ higher than the median of the overall functionality scale $(\mathrm{Mdn}=4)$. As for ExpQ, a satisfactory level of the sense of presence in the VE has been achieved $(M=5.20, \mathrm{SD}=0.94)$, which is above the median of the overall scale $(\mathrm{Mdn}=4)$. A Mann-Whitney U test revealed that the level of VR illusions was similar in both groups, as there was no significant difference between the sense of presence between experimental and control groups $(U=104.50, p=0.73)$. The same outcome applies to the sense of body ownership, as the sense of owning the virtual body of the avatar was marginally found to be the same for the two groups $(U=67.50, p=0.06)$. Overall, there was a positive correlation between presence and emotional impact, $r(28)=0.39, p=0.03$.

The only difference found between males and females concerned empathy. There was a significant difference between the dispositional empathy (IRI) between males $(M=3.36, \mathrm{SD}=0.23)$ and females $(M=3.72, \mathrm{SD}=0.30)$, $t(28)=-3.65, p=0.001$. No significant differences were

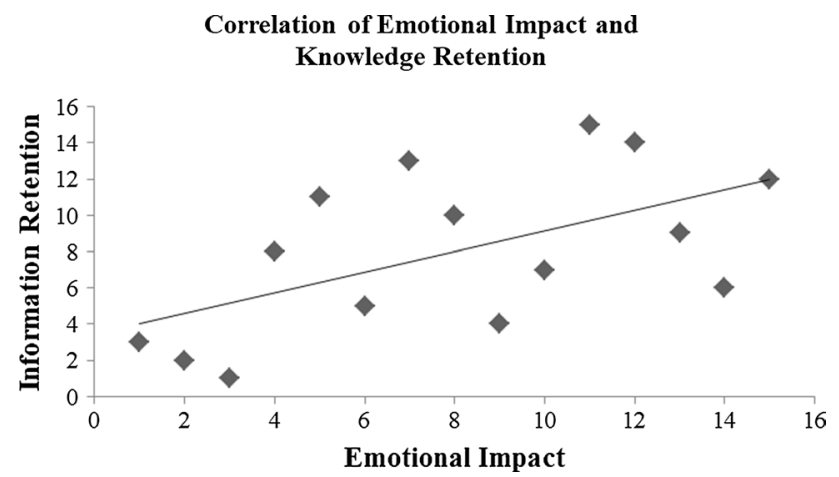

Fig. 4 Emotional impact is positively correlated with the post-test score on MDD. The correlation applies only to the group which experienced the positive ending found between males and females on presence, body ownership, knowledge retention, or emotional impact.

The qualitative analysis of the open questions revealed that some participants, who perceived the experience as "unpleasant," felt a distressing emotional "suffocation," regardless of experiencing the happy ending in the end, even though these reports were scarce. Other participants reported feeling overwhelmed while coming across the reflection of their depressed avatar through the mirror, which indicates that the mirror worked as intended by enhancing components of body ownership. Overall, an increase of "sadness" is evident to almost every participant after the experiment (in both, the experimental and control, groups) but those who already had a high level of sadness prior to the experiment were most likely to claim to have an unpleasant experience. Drastic fluctuations are recorded only for the affect labeled as "sadness."

\section{Discussion}

The findings of this research suggest that higher motivation, incited through the simulation with the positive ending, contributed to the stimulation of learning-related cognitive processes. The scenario of the experimental group, which included both the depressing and the "anti-depressing" scene (positive ending), was found to be more beneficial, from an educational standpoint, than the experience which contained the depressing scene only, even though by a small margin. Emotional impact is found to be positively correlated with a significant degree with the knowledge retention for the participants who strived to experience the positive ending. On the other hand, emotional impact that was incited without any motivation, through the purely negative simulation, showed only a trivial connection to knowledge retention. The bottom line of this finding is that the experimental group, in which motivational intensity was instigated, retained information comparable to the degree in which the affective states were triggered.

The first hypothesis concerned the effect of motivational intensity on emotional impact. Even though no noteworthy difference in emotional impact was found between the two groups, another interesting result was found regarding the affective states (DEQ). The correlations between dispositional empathy and affective states provide a strong predictive validity for psychological defense mechanisms that elucidate the cognitive behavior of empathic individuals. Before being exposed to the VE, the participants of the control group indicated that their affective states and dispositional empathy had a proportionally positive relationship, while, for the experimental group, the affective states of participants were found to be irrelevant with dispositional empathy. This result was completely subverted after the exposure to 
the VE, where the participants who were exposed to the purely negative scenario (control group) showed a trivial relationship between their affective states and dispositional empathy, while the participants who experienced the positive ending (experimental group) showed that the two gained a proportionally positive and robust relationship. This sudden change indicates that empathic disposition could be responsible for an aversion toward the purely depressive scenario and a tendency for emotional investment toward the other scenario. Cameron et al. argue that exposing oneself in situations where empathy is elicited could be emotionally taxing to the point where the mind incites an avoidant behavior (2019). It is speculated that Cameron and colleagues provide a plausible explanation for the aversion of the control group and the emotional reactivity of the experimental group. The participants who were encouraged to view the simulation as a challenge of overcoming a difficult situation (experimental group) were more likely to allow themselves being emotionally available during the simulated depressive episode because the premise of the situation implied a rewarding return of emotional investment. This reward was delivered in the form of the positive ending.

The second hypothesis concerns motivational intensity and knowledge retention, which are instigated by a narrative that could be characterized as unusual for an educational simulation. The narrative of the simulation is promoting a dejected mood of negative valence and arousal for the most part (if not always) in both instances of the simulation. The motivational intensity, which was administered on a different level in each group, seemed to contribute only a little in increasing knowledge retention. However, other factors, such as the fact that participants were already educated on MDD, played an important role in this outcome. Contrary to traditional beliefs on the valence of affective states, the negative valence of the narrative per se is found to be insignificant to knowledge retention. This outcome is consistent with reports of negative valence and low arousal experiences being neutral for cognitive abilities, such as memorizing and paying attention (Bradley et al. 1992; Christianson 1992; Libkuman et al. 2004). Bradley et al. highlight that folklore fallaciously encourages the idea that valence is closely related to memory performance (1992). However, throughout the literature, relations regarding affects and cognition remain ambiguous (Libkuman et al. 2004). The present study supports Bradley's findings on valence and cognitive functions while leaving room for further research.

Concerning the third hypothesis, results showed that motivational intensity through the narrative of the MDD simulation and the studied VR illusions (presence and body ownership) have no evident differences between experimental and control conditions, contrary to the results of other studies mentioned, such as those of Jun et al. (2018). Diemer et al. (2015) would argue that the reason for this outcome is that the two conditions did not differ significantly in emotional impact and indeed that should be the case because presence and emotional impact positively correlate overall, as shown by the statistical analysis. Regarding body ownership, it should be mentioned that the studies of Jun et al. refer to body ownership as "full-body ownership," suggesting that full-body synchronicity techniques, using a motion tracker suit, have been deployed. The kinesthetic enhancement of movement synchronization between user and avatar affects the sense of "embodiment" (Lopez et al. 2008), and this technique was different than the practice used in the present study. Regardless, Ma and Hommel stress that affective responses could be irrelevant to the sense of "body ownership" as this seems to be the case for the present study too (2013). The conclusion of Ma and Hommel derives from the evocation of fear-oriented affects, such as the affect caused by the threat of a knife targeting a hand. Even without the illusion of owning a targeted (by knife) hand of an agent being in effect, strong affective responses were still evident during an attack (Ma and Hommel 2013). The present study is consistent with the findings of previous research studies which support that affective states and body ownership, as well as presence, are loosely related.

In retrospect to the developed application, alternative implementation approaches of narratives on MDD have been considered. Even though the version of the positive ending is deemed to be the most effective of the two versions, in terms of both experience and educational capacity, there were instances where the positive ending seemed insufficient as a rewarding compensation for the "depressing" part. The prospect on which the MDD simulation was built was to create a plausible and accurate representation of the MDD symptomatology, and this milestone was achieved to a satisfactory degree, as the feedback of the participants suggested. The downside is to this is that most of the represented symptoms were represented too rigidly in regard to reality and this fact weighed heavily upon the "playful" aspect of the game. As Dunlap suggests, "Three-Dimensional representations [of mental illness] do not need to be true to life in terms of narrative or design, but they do need to reflect authentic experiences" (2018). It is assumed that if the design of the MDD simulation was more focused on the feeling of MDD instead of how it ostensibly is to be affected by MDD, then the experience could have been more entertaining and thus even more effective as a learning tool.

Lastly, it was once again validated that females tend to score higher on the IRI test than males (Davis 1980) but this study did not point toward any other drastic differences between males and females in the sense of VR presence, body ownership, emotional impact, or information retention. The avatar that the stereotype lift elicited by the female avatar took place during the VR experience and equalized the empathy of participants, which is an ideal outcome since 
that was the aim of using a female avatar (Walton and Cohen 2003), as is elaborated in section "The avatar".

\section{Research limitations}

A self-evaluation of the methodology and the general direction of this research has also been applied. The application could make better use of the interaction between the user and the virtual environment. The interactivity was limited to the initiation of certain events that mostly revolved around the triggering of voice clips. All of the events were autonomous and had no consequences to other events, except for the positive ending, which required the activation of all the other points of interest. The limited interactivity was practically sufficient, as the functionality scale indicated, but more complex interactive sequences could certainly induce a more game-like experience. Admittedly, the degree of agency over the progression of the narrative is important for the production of meaning (Tanenbaum and Tanenbaum 2010). Additionally, some minor caveats greatly affected the outcome of the study. First and foremost, the majority of participants happened to be quite knowledgeable regarding depressive disorders, so despite the fact that there was an increase of knowledge on MDD followed by the use of the application, the full extent of the application's educational capability remains unclear. Secondly, the average time of completing the required tasks should have been measured during the experiment. It was observed that the group which had a goal, namely the anticipation of an incident after completing the required tasks (i.e., the experimental group), was able to locate the points of interest much faster than the other group (i.e., the control group). However, this claim, albeit reasonable, is unfounded since the required measure of time was excluded from the study. The aspect of completion time could have brought to light some interesting information about the experience of using the MDD simulator. Finally, the findings of the study relied heavily on the interpretations of participants because of the methodological tools used. Given the fact that the empathy of females was found to be significantly higher than the empathy of males, it could be argued that the lack of differences between the emotional impact of males and females is quite odd. This lack of differences despite the empathy dichotomy, even though plausible, could be the result of males overestimating their change in affective states (DEQ) or females underestimating the same measure or both. A study on feelings of depression and memory bias suggests that females tend to rate their negative emotions from within a span of a week as more negative than the average of their daily ratings of the same emotions (Urban et al. 2018), which shows the precariousness of rating negative affects from memory.

\section{Conclusions}

The created educational simulation on MDD is found to be successful in terms of educational capacity. However, the fact that most of the participants were already informed about MDD interfered to a degree with investigating the link between motivational intensity and knowledge retention. The two versions of the simulation were found to have little differences in the aspects that have been investigated, with the most important difference being a positive correlation between emotional impact and knowledge retention for the experimental group. Also, the utilization of motivational intensity was found to mostly affect empathic individuals. The bibliography concerning the study of simulations and their emergent narratives remains widely unexplored. As G. Frasca states "Sadly, our current knowledge of simulation rhetoric is extremely limited" (2013). Hopefully, this research could provide some insight for future research on simulations and game narratives, while stressing that the emotional impact, as a result of perceived change in affective states, plays a prominent role in the educational aspect of serious games. Following the example of the MDD VR simulator, similar applications that use narrative as a vehicle for mediating experiences of other mental conditions or social issues could be leveraged for carrying out similar studies and adding up to the present findings.

Future studies could be benefitted by the findings of this study by taking into consideration that the link between emotional impact and knowledge retention could be used for improving the educational efficacy of simulations. Even so, it is highlighted that factors, other than affective states, are also important for the stimulation of knowledge retention. Some of these factors are technically oriented, as they relate to experience design and the integration of the learning material to the MDD simulation. For instance, it is imperative to keep in mind that motivational intensity affects the interplay between emotional impact and knowledge retention on a notable degree. This conclusion stems from the result that knowledge retention is positively and significantly correlated with emotional impact but only for the group of participants who experience the positive ending. Moreover, future studies may use the present application to focus on motivational intensity and cognitive scope, which are aspects of affective states which have been omitted from the present study. The cognitive scope encompasses the measurements of perception, attention, categorization, and memory-related tasks (Summerell et al. 2019). The presented application certainly induces sadness, even though limited to "sensitive on negative valence" individuals, which could be further leveraged for studies on the dynamic between motivational intensity and 
cognitive scope. Research on the said dynamic is worth conducting because the broadening of the cognitive scope encourages the increase of motivation and the identification of goal opportunities (Harmon-Jones et al. 2012). Furthermore, the application could be used for the study of raising awareness toward healthy individuals, such as individuals from specific ethnic groups, who display biases toward the importance of acknowledging sufferers of MDD as clinical patients (Brown et al. 2011).

Supplementary Information The online version contains supplementary material available at https://doi.org/10.1007/s10055-021-00568-5.

Open Access This article is licensed under a Creative Commons Attribution 4.0 International License, which permits use, sharing, adaptation, distribution and reproduction in any medium or format, as long as you give appropriate credit to the original author(s) and the source, provide a link to the Creative Commons licence, and indicate if changes were made. The images or other third party material in this article are included in the article's Creative Commons licence, unless indicated otherwise in a credit line to the material. If material is not included in the article's Creative Commons licence and your intended use is not permitted by statutory regulation or exceeds the permitted use, you will need to obtain permission directly from the copyright holder. To view a copy of this licence, visit http://creativecommons.org/licenses/by/4.0/.

\section{References}

American Psychiatric Association (2013) Diagnostic and statistical manual of mental disorders (DSM-5®). American Psychiatric Publisher

AShamaluevMusic. (2017, December 08). Need instrumental background music? [Internet]. https://www.ashamaluevmusic.com/. Accessed 10 Apr 2019

Banakou D, Hanumanthu PD, Slater M (2016) Virtual embodiment of white people in a black virtual body leads to a sustained reduction in their implicit racial bias. Front Hum Neurosci 10:601

Baños RM, Botella C, Alcañiz M, Liaño V, Guerrero B, Rey B (2004) Immersion and emotion: their impact on the sense of presence. Cyberpsychol Behav 7(6):734-741

Baños RM, Botella C, Rubió I, Quero S, García-Palacios A, Alcañiz M (2008) Presence and emotions in virtual environments: the influence of stereoscopy. Cyberpsychol Behav 11(1):1-8

Baron-Cohen S (2002) The extreme male brain theory of autism. Trends Cogn Sci 6(6):248-254

Barrett LF (1998) Discrete emotions or dimensions? The role of valence focus and arousal focus. Cogn Emot 12(4):579-599

Bazalgette C, Buckingham D (2013) Literacy, media and multimodality: A critical response. Literacy 47(2):95-102

Beck AT, Alford BA (2009) Depression: causes and treatment. University of Pennsylvania Press, Pennsylvania

Bizzocchi J, Tanenbaum J (2012) Mass Effect 2: A case study in the design of game narrative. Bull Sci Technol Soc 32(5):393-404

Bower GH, Gilligan SG, Monteiro KP (1981) Selectivity of learning caused by affective states. J Exp Psychol Gen 110(4):451

Bradley MM, Codispoti M, Cuthbert BN, Lang PJ (2001) Emotion and motivation I: defensive and appetitive reactions in picture processing. Emotion 1(3):276
Bradley MM, Greenwald MK, Petry MC, Lang PJ (1992) Remembering pictures: pleasure and arousal in memory. J Exp Psychol Learn Mem Cogn 18(2):379

Brodal P (2004) The central nervous system: structure and function. Oxford University Press, Oxford

Brown JS, Casey SJ, Bishop AJ, Prytys M, Whittinger N, Weinman J (2011) How black African and white British women perceive depression and help-seeking: a pilot vignette study. Int J Soc Psychiatry 57(4):362-374

Caligiuri MP, Ellwanger J (2000) Motor and cognitive aspects of motor retardation in depression. J Affect Disord 57(1-3):83-93

Cameron CD, Hutcherson CA, Ferguson AM, Scheffer JA, Hadjiandreou E, Inzlicht M (2019) Empathy is hard work: People choose to avoid empathy because of its cognitive costs. J Exp Psychol Gen 148(6):962

Chittaro L, Buttussi F, Zangrando N (2014, November) Desktop virtual reality for emergency preparedness: user evaluation of an aircraft ditching experience under different fear arousal conditions. In: Proceedings of the 20th ACM symposium on virtual reality software and technology, pp. 141-150

Christianson SÅ (1992) Emotional stress and eyewitness memory: a critical review. Psychol Bull 112(2):284

Christofi M, Michael-Grigoriou D, Kyrlitsias C (2020) A virtual reality simulation of drug users' everyday life: the effect of supported sensorimotor contingencies on empathy. Front Psychol 11:1242

Consalvo M (2003) Zelda 64 and video game fans: a walkthrough of games, intertextuality, and narrative. Television \& New Media 4(3):321-334

Davis MH (1980) A multidimensional approach to individual differences in empathy. JSAS Catalog of Selected Documents in Psychology 10:85.

Davis MH (1983) Measuring individual differences in empathy: Evidence for a multidimensional approach. J Pers Soc Psychol 44(1): 113

Decety J, Hodges SD (2006) The social neuroscience of empathy. In: Van Lange PAM (ed) Bridging social psychology: Benefits of transdisciplinary approaches. Lawrence Erlbaum Associates Publishers, pp 103-109

Diemer J, Alpers GW, Peperkorn HM, Shiban Y, Mühlberger A (2015) The impact of perception and presence on emotional reactions: a review of research in virtual reality. Front Psychol $6: 26$

Dunlap KN (2018) Representation of mental illness in video games. In: Proceedings of the 2018 connected learning summit, Massachusetts institute of technology, pp 77-86

Ekman P (1992) An argument for basic emotions. Cogn Emot 6(3-4):169-200

Feldman LA (1995) Valence focus and arousal focus: Individual differences in the structure of affective experience. J Pers Soc Psychol 69(1):153

Fine C (2008) Will working mothers' brains explode? The popular new genre of neurosexism. Neuroethics 1(1):69-72

Frasca G (2013) Simulation versus narrative: introduction to ludology. In: The video game theory reader. Routledge, pp 243-258

Freeman J, Lessiter J, Pugh K, Keogh E (2005, September) When presence and emotion are related, and when they are not. In: Proceedings of the 8th annual international workshop on presence (PRESENCE 2005). International Society for Presence Research, pp 213-219

Freina L, Ott M (2015, April) A literature review on immersive virtual reality in education: state of the art and perspectives. In: The International Scientific Conference eLearning and Software for Education, vol 1, no 133, pp 10-1007

Gallagher S (2012) Empathy, simulation, and narrative. Sci Context 25(3):355-381 
Green MJ, Myers KR (2010) Graphic medicine: use of comics in medical education and patient care. BMJ 340:c863. https://doi.org/10. 1136/bmj.c863

Hadjipanayi C, Michael-Grigoriou D (2020) Conceptual knowledge and sensitization on Asperger's syndrome based on the constructivist approach through virtual reality. Heliyon 6(6):e04145

Hammond HK (2009) Graphic novels and multimodal literacy: a reader response study. Retrieved from the University of Minnesota Digital Conservancy. https://hdl.handle.net/11299/48560

Harmon-Jones C, Bastian B, Harmon-Jones E (2016) The discrete emotions questionnaire: a new tool for measuring state self-reported emotions. PLoS ONE 11(8):e0159915. https://doi.org/10.1371/ journal.pone.0159915

Harmon-Jones E, Gable P, Price TF (2012) The influence of affective states varying in motivational intensity on cognitive scope. Front Integr Neurosci 6:73

Harmon-Jones E, Gable PA, Price TF (2013) Does negative affect always narrow and positive affect always broaden the mind? Considering the influence of motivational intensity on cognitive scope. Curr Dir Psychol Sci 22(4):301-307

Hasler BS, Spanlang B, Slater M (2017) Virtual race transformation reverses racial in-group bias. PLoS ONE 12(4):e0174965. https:// doi.org/10.1371/journal.pone.0174965

Highet NJ, Hickie IB, Davenport TA (2002) Monitoring awareness of and attitudes to depression in Australia. Med J Aust 176(10):S63-S63

Hudson DL, Eaton J, Banks A, Sewell W, Neighbors H (2018) "Down in the Sewers": Perceptions of depression and depression care among African American men. Am J Mens Health 12(1):126-137

Izard CE (1992). Basic emotions, relations among emotions, and emotion-cognition relations. Psychological Review 99(3):561-565

Johnston WA, Dark VJ (1986) Selective attention. Annu Rev Psychol 37(1):43-75

Jun J, Jung M, Kim SY, Kim K (2018, April). Full-body ownership illusion can change our emotion. In: Proceedings of the $2018 \mathrm{CHI}$ conference on human factors in computing systems, pp 1-11

Kilteni K, Groten R, Slater M (2012) The sense of embodiment in virtual reality. Presence Teleop Virtual Environ 21(4):373-387

Kiropoulos LA, Griffiths KM, Blashki G (2011) Effects of a multilingual information website intervention on the levels of depression literacy and depression-related stigma in Greek-born and Italianborn immigrants living in Australia: a randomized controlled trial. Journal of medical Internet research 13(2):e34

Kress GR (2010) Multimodality: a social semiotic approach to contemporary communication. Taylor \& Francis, London

Lauber C, Falcato L, Nordt C, Rössler W (2003) Lay beliefs about causes of depression. Acta Psychiatr Scand 108:96-99

Libkuman T, Stabler C, Otani H (2004) Arousal, valence, and memory for detail. Memory 12(2):237-247

Lopez C, Halje P, Blanke O (2008) Body ownership and embodiment: vestibular and multisensory mechanisms. Clin Neurophysiol 38(3):149-161

Lee JER, Nass CI, Bailenson JN (2014) Does the mask govern the mind?: Effects of arbitrary gender representation on quantitative task performance in avatar-represented virtual groups. Cyberpsychol Behav Soc Netw 17(4):248-254

Maister L, Slater M, Sanchez-Vives MV, Tsakiris M (2015) Changing bodies changes minds: owning another body affects social cognition. Trends Cogn Sci 19(1):6-12

Ma K, Hommel B (2013) The virtual-hand illusion: effects of impact and threat on perceived ownership and affective resonance. Front Psychol 4:604

Marsh R, Hao X, Xu D, Wang Z, Duan Y, Liu J, Yu S (2010) A virtual reality-based FMRI study of reward-based spatial learning. Neuropsychologia 48(10):2912-2921
Moser C, Fang X (2015) Narrative structure and player experience in role-playing games. Int J Hum Comput Interact 31(2):146-156

Muller DA, Van Kessel CR, Janssen S (2017, October). Through Pink and Blue glasses: designing a dispositional empathy game using gender stereotypes and Virtual Reality. In: Extended abstracts publication of the annual symposium on computer-human interaction in play, pp 599-605

Oculus (n.d.) Notes on blindness [Internet]. Retrieved from: https:// oculus.com/experiences/gear-vr/1015802351839289/.

O'Sullivan J (2019) Interactivity and the illusion of choice. In: Towards a digital poetics. Palgrave Macmillan, Cham, pp 77-93

Panksepp J (2004) Affective neuroscience: the foundations of human and animal emotions. Oxford University Press, Oxford

Qin H, Patrick Rau PL, Salvendy G (2009) Measuring player immersion in the computer game narrative. Int J Hum Comput Interact 25(2):107-133

Rao N, Chu SL, Faris RW, Ospina D (2019, November) The effects of interactive emotional priming on storytelling: An exploratory study. In: International conference on interactive digital storytelling. Springer, Cham, pp. 395-404

Richter M (2013) A closer look into the multi-layer structure of motivational intensity theory. Soc Pers Psychol Compass 7(1):1-12

Riva G, Mantovani F, Capideville CS, Preziosa A, Morganti F, Villani D, Alcañiz M (2007) Affective interactions using virtual reality: the link between presence and emotions. Cyberpsychol Behav 10(1):45-56

Ryan ML (2003) Narrative as virtual reality: immersion and interactivity in literature and electronic media. J Aesthetics Art Criticism 61(2):206-207

Salen K, Zimmerman E (2004) Rules of play: Game design fundamentals. MIT Press

Seth AK, Suzuki K, Critchley HD (2012) An interoceptive predictive coding model of conscious presence. Front Psychol 2:395

Schott, G. R. (2017). That Dragon, Cancer: contemplating life and death in a medium that has frequently trivialized both. In: Digital Games Research Association Conference (DiGRA), vol 14, no 1. Digital Games Research Association, pp 1-10

Schimelpfening N (2018, August 21). Grief vs. depression: which is it? [Internet]. https://www.verywellmind.com/grief-and-depre ssion-1067237.

Schimmenti A, Jonason PK, Passanisi A, La Marca L, Di Dio N, Gervasi AM (2019) Exploring the dark side of personality: emotional awareness, empathy, and the dark triad traits in an Italian sample. Curr Psychol 38(1):100-109

Schultheis MT, Rizzo AA (2001) The application of virtual reality technology in rehabilitation. Rehabil Psychol 46(3):296

Slater M, Usoh M (1993, September). Presence in immersive virtual environments. In: Proceedings of IEEE virtual reality annual international symposium. IEEE, pp 90-96

Slater M (1999) Measuring presence: A response to the Witmer and Singer presence questionnaire. Presence 8(5):560-565

Slater M, Steed A (2000) A virtual presence counter. Presence Teleoperators Virtual Environ 9(5):413-434

Slater M (2003) A note on presence terminology. Presence Connect $3(3): 1-5$

Slater M (2009) Place illusion and plausibility can lead to realistic behaviour in immersive virtual environments. Philos Trans R Soc B Biol Sci 364(1535):3549-3557

Steed A, Pan Y, Watson Z, Slater M (2018) "We Wait"-The impact of character responsiveness and self embodiment on presence and interest in an immersive news experience. Front Robot AI 5:112

Summerell E, Harmon-Jones C, Kelley NJ, Peterson CK, KrstanoskaBlazeska K, Harmon-Jones E (2019) Does cognitive broadening reduce anger? Front Psychol 9:2665 
Tanenbaum K, Tanenbaum TJ (2010) Agency as commitment to meaning: communicative competence in games. Digit Creat 21(1):1117. https://doi.org/10.1080/14626261003654509

That Dragon, Cancer. (2016). That Dragon, Cancer [Internet]. Retrieved from: http://www.thatdragoncancer.com/.

Tsitsas G, Theodosopoulou M, Malikiossi-Loizou M (2012) Interpersonal reactivity scale. In: Stalikas A, Triliva S, Roussou P (eds)

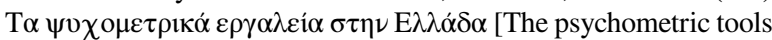
in Greece]. Pedio, Athens

Unity Asset Store (2017, August 17) Jimmy Gao-Asset Store [Internet]. https://assetstore.unity.com/publishers/30349. Accessed 10 Apr 2019

Urban EJ, Charles ST, Levine LJ, Almeida DM (2018) Depression history and memory bias for specific daily emotions. PloS One 13(9):e0203574
Villani D, Lucchetta M, Preziosa A, Riva G (2009) The role of interactive media features on the affective response: a virtual reality study. Int J Hum Comput Interact 1(5):1-21

Walton GM, Cohen GL (2003) Stereotype lift. J Exp Soc Psychol 39(5):456-467

Publisher's Note Springer Nature remains neutral with regard to jurisdictional claims in published maps and institutional affiliations. 